\title{
Analysis of Yield Components and Aroma of Small Grain Aromatic Rice (Oryza sativa L.) in Bangladesh
}

\author{
Parth Sarothi Saha ${ }^{1}$, Mizan Ul Islam ${ }^{1}$, Md. Sirajul Islam² and Muhammad Abdus Salam ${ }^{1}$ \\ ${ }^{1}$ BRAC Agricultural Research and Development Centre, CERDI Road, Gazipur-1701, Bangladesh; \\ ${ }^{2}$ Agriculture and Food Security Program, BRAC, Dhaka, Bangladesh \\ *Corresponding author and Email: parth.saha@brac.net
}

Received: 4 May 2015

Accepted: 12 December 2015

\begin{abstract}
The study was conducted to evaluate the extent of variability among the small grain aromatic (SGA) rice (Oryza sativa L.) genotypes for yield and yield components. Twenty four popular SGA rice genotypes were evaluated for yield and yield contributing characters in BRAC Agricultural Research and Development Centre, Gazipur, Bangladesh. BRRI dhan34 was used as check variety. Highest grain yield per plant was observed in Chinikanai-1, which was followed by Kalijira PL-9, Kalijira PL3 and Badshabhog. Chinikanai-1 had the highest number of grains per panicle. Correlation analysis revealed that the number of panicles per plant $(r=0.646)$ and number of grains per panicle $(r=0.525)$ had the positive contribution to grain yield. Based on sensory test, it was found that 18 genotypes were scented and six were lightly scented. After evaluation of yield components, four genotypes namely Chinikanai-1, Kalijira PL-9, Kalijira PL-3 and Badshabhog were selected as outstanding genotypes, which can be used as potential breeding materials for sub-tropical environment of Bangladesh.
\end{abstract}

Keywords: Aromatic rice, grain yield, yield component, small grain rice

\section{Introduction}

Aromatic rice (Oryza sativa L.) is preferred by consumers all over the world due to its flavor and palatability. Small grain aromatic (SGA) rice has been the chief ingredients for preparation of many fabulous dishes in Bangladesh and Eastern India. The important feature of this group of rice is its palatable aroma which has been considered as a high value trait for rice. Grain quality of rice plays an important role in consumers' acceptability and after yield, it is the second major breeding consideration (Jewel et al., 2011). Low yield is a common character of aromatic rice and consequently rice breeders are trying to develop the agronomic traits to gain better grain yield. The success stories on the improvement of SGA rice is very much limited except BRRI dhan34. The weaknesses are photoperiod sensitivity, poor yield potential, susceptibility to lodging, pests and diseases, and severe lodging (Golam et al., 2011). Agronomic value of rice depends on increase grain yield and its associated traits such as panicles per plant, grains per panicle, and thousand grain weight.

Genotypic evaluation of yield components has identified their relationship with grain yield and it is helpful to identify superior SGA genotype(s) for improvement (Tahir et al., 2002). Correlation analysis of characters can be used as tool for indirect selection. Correlation studies help the 
plant breeders during selection and provide an understanding of yield components. Therefore, this study was conducted to evaluate the extent of variability among the SGA rice genotypes for yield and its contributing traits.

\section{Materials and Methods}

Twenty four small grain aromatic rice genotypes including a standard check variety (BRRI dhan34) were grown at BRAC Agricultural Research and Development Centre (BARDC), Gazipur, Bangladesh during Aman season 2012. The experiment was laid out in a Randomized Complete Block Design with three replications. The climatic condition was hot and humid with frequent rain during vegetative phase, and cold and dry during the reproductive and ripening phase (Table 1).

Seeding was done on 05 August 2012 and 30 days old seedlings were transplanted. Twenty $\mathrm{cm}$ distance was maintained between the rows and $15 \mathrm{~cm}$ between the plants. Single seedling was used for transplanting and unit plot size was 3.24 $\mathrm{m}^{2}$. TSP and MoP were applied at the rate of 100 $\mathrm{Kg} / \mathrm{ha}$ and $60 \mathrm{Kg} / \mathrm{ha}$, respectively during final land preparation. Urea was applied at the rate of $120 \mathrm{Kg} / \mathrm{ha}$ in two splits at 10 and 30 days after transplanting. A hand weeding was done at 25 days after transplanting.

Data on days to $50 \%$ flowering was recorded. Five plants were randomly selected excluding border rows and any missing plants in a plot for data on plant height $(\mathrm{cm})$, tillers per plant, panicle per plant, panicle length $(\mathrm{cm})$, grains per panicle, sterility percentage, thousand grain weight $(\mathrm{g})$, spikelet length $(\mathrm{mm})$, spikelet breadth $(\mathrm{mm})$ and grain yield per plant $(\mathrm{g})$. Thousand grain weight and grain yield per plant were adjusted to $14 \%$ moisture.

Grain aroma was assessed by a panel consisting of three members following the procedure described in the Thirteenth International Fine Grain Aromatic Rice Observational Nursery of the International Network for Genetic Evaluation of Rice (INGER) coordinated by IRRI (IRFAON, 2012). In brief, few grains were dehulled, then carefully crushed between the teeth and inhaled the smell slowly to detect the presence and intensity of aroma. Then all the SGA varieties were scored for aroma intensity based on the scale of the Standard Evaluation System for Rice (IRRI, 2002).

Data were analyzed using PLBSTAT Version 2N (Utz, 2007) and Microsoft Excel. Analysis of variance was used to test the significance of variance sources, while DMRT test $(p=0.05)$ was employed to compare the differences among treatment means. The correlation coefficient analysis was conducted to find the relationship of different traits.

\section{Results and Discussion}

\subsection{Plant height}

Plant height varied significantly among the genotypes (Table 2). Coefficient of variation for plant height was $3.53 \%$ (Table 2). Similar result was reported by Kole and Hasib (2008). Plant height ranged between 92 to $133 \mathrm{~cm}$ (Table 3). Robin and Saha (2015) recorded slightly higher plant height for Kalijira $(127 \mathrm{~cm})$. This is possibly due to late seeding of the SGA genotypes. Minimum plant height was recorded in Sakkorkhora, while maximum height was observed in Chinikanai-1.

\subsection{Number of tillers per plant}

Genetic differences were significant at $1 \%$ level among rice genotypes in respect of number of tillers per plant (Table 2). Coefficient of variation for number of tillers per plant was $20.09 \%$ (Table 2). The number of tillers per plant ranged from 7 to 14 (Table 3). Minimum and maximum number of tillers was observed in Begun bichi and Tilkapur, respectively.

\subsection{Number of panicles per plant}

There were significant variations among the genotypes for number of panicles per plant (Table 2). Coefficient of variation for number of panicles per plant was $20.84 \%$ (Table 2). 
Table 1. Meteorological data recorded at the experimental site during the study period

\begin{tabular}{|c|c|c|c|c|c|c|c|c|}
\hline \multirow[t]{2}{*}{ Month } & \multicolumn{2}{|c|}{ Air temp (\%) } & \multirow[t]{2}{*}{ Rainfall (mm) } & \multicolumn{2}{|c|}{ Relative humidity (\%) } & \multirow{2}{*}{$\begin{array}{l}\text { Sunshine } \\
\text { (hrs/day) }\end{array}$} & \multirow[t]{2}{*}{ Cloudy (hrs/day) } & \multirow{2}{*}{$\begin{array}{l}\text { Solar radiation } \\
\left(\mathrm{gm} / \mathrm{cal} / \mathrm{cm}^{2} / \text { day }\right)\end{array}$} \\
\hline & Max & Min & & $9 \mathrm{am}$ & $2 \mathrm{pm}$ & & & \\
\hline Aug-2012 & 32.4 & 26.7 & 18.18 & 85.39 & 73.72 & 5.80 & 7.10 & 351.14 \\
\hline Sep-2012 & 32.4 & 26.9 & 15.51 & 84.13 & 77.53 & 4.15 & 8.05 & 276.94 \\
\hline Oct-2012 & 31.9 & 24.0 & 0.73 & 78.06 & 64.29 & 6.70 & 4.79 & 337.78 \\
\hline Nov-2012 & 28.4 & 18. 6 & 2.39 & 76.07 & 57.63 & 7.08 & 4.29 & 292.18 \\
\hline Dec-2012 & 23.7 & 13.7 & 0.12 & 90.13 & 65.52 & 4.43 & 6.17 & 216.17 \\
\hline
\end{tabular}

Table 2. Genotypic variations of agronomic traits in 24 SGA rice genotypes of Bangladesh

\begin{tabular}{|c|c|c|c|c|c|c|c|c|c|c|c|c|}
\hline $\begin{array}{l}\text { Source of } \\
\text { variation }\end{array}$ & $\mathrm{DF}$ & GYP & $\mathrm{PH}$ & TILL & PN & PL & $50 \mathrm{~F}$ & $\mathrm{G}$ & $\mathrm{S}(\%)$ & TGW & SL & SB \\
\hline Genotype & 23 & $37.99^{* * 2}$ & $266.93^{* *}$ & $11.96^{* *}$ & $10.78^{* * *}$ & $8.06^{* *}$ & $47.01^{* *}$ & $3352.32^{* *}$ & $117.11^{* \cdots}$ & $0.800^{* * *}$ & $0.49^{* * *}$ & $0.13^{* *}$ \\
\hline Replication & 2 & $27.43^{\mathrm{NS}}$ & $26.01^{\mathrm{NS}}$ & $12.60^{\mathrm{NS}}$ & $6.29^{\mathrm{NS}}$ & $7.10^{\mathrm{NS}}$ & $5.10^{\mathrm{NS}}$ & $643.76^{\mathrm{NS}}$ & $2.20^{\mathrm{NS}}$ & $0.004^{\mathrm{NS}}$ & $0.05^{\mathrm{NS}}$ & $0.01^{\mathrm{NS}}$ \\
\hline Error & 46 & 10.73 & 15.88 & 4.51 & 3.68 & 2.47 & 0.47 & 495.59 & 31.32 & 0.004 & 0.09 & 0.02 \\
\hline Mean & & 15.20 & 113.03 & 11.00 & 9.00 & 24.15 & 78.31 & 155.00 & 15.16 & 11.00 & 6.11 & 2.28 \\
\hline $\mathrm{CV}(\%)$ & & 21.56 & 3.53 & 20.09 & 20.84 & 6.51 & 0.88 & 14.32 & 36.92 & 1.830 & 5.00 & 5.96 \\
\hline
\end{tabular}

** indicate significantly different at $1 \%$, NS: not significant. Indicators: GYP=grain yield per plant $(\mathrm{g})$, PH=plant height, TILL=tillers per plant, $\mathrm{PN}=$ panicles per plant, $50 \mathrm{~F}=$ days to $50 \%$ flowering, $\mathrm{PL}=$ panicle length, $\mathrm{G}=$ grains per panicle, $\mathrm{S}(\%)=$ grain sterility $(\%)$, TGW=thousand grain weight $(\mathrm{g})$,

$\mathrm{SL}=$ spikelet length and $\mathrm{SB}=$ spikelet breadth 
Table 3. Mean comparison of different traits the genotypes through Duncan Multiple Range Test (DMRT)

\begin{tabular}{|c|c|c|c|c|c|c|c|c|c|c|c|}
\hline Genotypes & GYP (g) & $\mathrm{PH}(\mathrm{cm})$ & $\begin{array}{l}\text { TILL } \\
\text { (no.) }\end{array}$ & PN & $\mathrm{PL}(\mathrm{cm})$ & $50 \mathrm{~F}$ & G & $\mathrm{S}(\%)$ & TGW $(\mathrm{g})$ & $\mathrm{SL}(\mathrm{mm})$ & SB \\
\hline Chinikanai-1 & $21.24 \mathrm{a}$ & $133.00 \mathrm{a}$ & $13 \mathrm{ab}$ & $9 \mathrm{a}-\mathrm{f}$ & $26.00 \mathrm{a}-\mathrm{c}$ & $80 \mathrm{de}$ & $219 a$ & $08.01 \mathrm{e}$ & $10.8 \mathrm{~g}$ & $5.96 \mathrm{~b}-\mathrm{f}$ & $2.17 \mathrm{e}-\mathrm{i}$ \\
\hline KalijiraPL-9 & 19.96ab & $110.00 \mathrm{e}-\mathrm{i}$ & $9 \mathrm{~b}-\mathrm{e}$ & $9 \mathrm{a}-\mathrm{f}$ & $22.67 \mathrm{~d}-\mathrm{f}$ & $79 \mathrm{ef}$ & $203 \mathrm{ab}$ & $11.23 \mathrm{de}$ & $11.1 \mathrm{fg}$ & $6.29 \mathrm{~b}-\mathrm{e}$ & $2.51 b c$ \\
\hline Kalijira PL-3 & $19.30 \mathrm{a}-\mathrm{c}$ & $113.33 \mathrm{c}-\mathrm{h}$ & $12 \mathrm{a}-\mathrm{d}$ & $11 \mathrm{a}-\mathrm{c}$ & $24.00 \mathrm{~b}-\mathrm{f}$ & $77 \mathrm{gh}$ & $167 \mathrm{~b}-\mathrm{d}$ & $13.63 \mathrm{c}-\mathrm{e}$ & $10.3 \mathrm{hi}$ & $6.11 \mathrm{~b}-\mathrm{f}$ & $2.45 \mathrm{~b}-\mathrm{d}$ \\
\hline Badshabhog & $19.18 \mathrm{a}-\mathrm{c}$ & $112.67 \mathrm{c}-\mathrm{h}$ & $13 \mathrm{a}-\mathrm{c}$ & $12 \mathrm{a}$ & $25.33 \mathrm{a}-\mathrm{d}$ & $79 \mathrm{f}$ & $159 \mathrm{~cd}$ & $11.78 \mathrm{de}$ & $9.8 \mathrm{jk}$ & $5.78 c-f$ & $2.43 \mathrm{~b}-\mathrm{e}$ \\
\hline BRRI dhan34 & $17.25 \mathrm{a}-\mathrm{d}$ & $107.00 \mathrm{~g}-\mathrm{i}$ & 10 a-e & 10 a-e & $25.00 \mathrm{a}-\mathrm{d}$ & $77 \mathrm{~h}$ & $177 \mathrm{bc}$ & $19.37 \mathrm{~b}-\mathrm{d}$ & $10.0 \mathrm{ij}$ & $5.89 \mathrm{~b}-\mathrm{f}$ & $1.98 \mathrm{i}$ \\
\hline Begun bichi & $07.80 \mathrm{~g}$ & $115.67 \mathrm{c}-\mathrm{f}$ & $7 \mathrm{e}$ & $7 \mathrm{~d}-\mathrm{f}$ & $21.00 \mathrm{f}$ & $71 \mathrm{kl}$ & $120 \mathrm{e}-\mathrm{g}$ & $23.54 \mathrm{a}-\mathrm{c}$ & $9.6 \mathrm{kl}$ & $5.71 \mathrm{~d}-\mathrm{f}$ & $2.1 \mathrm{~g}-\mathrm{i}$ \\
\hline Binnaful & $15.74 \mathrm{a}-\mathrm{e}$ & $103.33 \mathrm{i}$ & $9 \mathrm{~b}-\mathrm{e}$ & $9 \mathrm{a}-\mathrm{f}$ & $21.33 \mathrm{ef}$ & $82 \mathrm{bc}$ & $153 \mathrm{c}-\mathrm{e}$ & $19.24 \mathrm{~b}-\mathrm{d}$ & $11.9 \mathrm{~d}$ & $5.95 \mathrm{~b}-\mathrm{f}$ & $2.42 \mathrm{~b}-\mathrm{e}$ \\
\hline BR5 (Dulabhog) & $10.93 \mathrm{~d}-\mathrm{g}$ & $107.33 \mathrm{~g}-\mathrm{i}$ & $8 \mathrm{de}$ & $8 b-f$ & $27.33 \mathrm{a}$ & $78 \mathrm{f}$ & $132 \mathrm{~d}-\mathrm{f}$ & $20.52 \mathrm{~b}-\mathrm{d}$ & $10.3 \mathrm{hi}$ & $6.38 \mathrm{bc}$ & $2.02 \mathrm{hi}$ \\
\hline Chinikanai-2 & $15.11 \mathrm{a}-\mathrm{f}$ & $126.00 \mathrm{~b}$ & $8 \mathrm{de}$ & $6 \mathrm{ef}$ & $25.00 \mathrm{a}-\mathrm{d}$ & $82 \mathrm{bc}$ & $201 \mathrm{ab}$ & $09.97 \mathrm{de}$ & $11.6 \mathrm{de}$ & $5.85 \mathrm{~b}-\mathrm{f}$ & $2.20 \mathrm{~d}-\mathrm{i}$ \\
\hline Chinigura & $14.15 \mathrm{~b}-\mathrm{f}$ & $114.67 \mathrm{c}-\mathrm{g}$ & 9 c-e & $8 \mathrm{c}-\mathrm{f}$ & $23.67 \mathrm{~b}-\mathrm{f}$ & $81 \mathrm{c}$ & $200 \mathrm{ab}$ & $16.98 \mathrm{~b}-\mathrm{e}$ & 9.41 & $5.94 b-f$ & $2.01 \mathrm{hi}$ \\
\hline Chinigura PL-2 & $14.19 b-f$ & $107.00 \mathrm{~g}-\mathrm{i}$ & $11 \mathrm{a}-\mathrm{e}$ & $9 \mathrm{a}-\mathrm{e}$ & $23.33 \mathrm{c}-\mathrm{f}$ & $81 \mathrm{~cd}$ & $159 \mathrm{~cd}$ & $06.74 \mathrm{e}$ & 9.41 & $5.87 \mathrm{~b}-\mathrm{f}$ & $1.97 \mathrm{i}$ \\
\hline Kalijira (Normal) & $18.47 \mathrm{a}-\mathrm{c}$ & $106.33 \mathrm{hi}$ & $12 \mathrm{a}-\mathrm{d}$ & $12 \mathrm{ab}$ & $24.67 \mathrm{a}-\mathrm{d}$ & $82 \mathrm{bc}$ & $180 \mathrm{bc}$ & $16.30 \mathrm{~b}-\mathrm{e}$ & $8.9 \mathrm{~m}$ & $6.27 \mathrm{~b}-\mathrm{e}$ & $2.26 \mathrm{c}-\mathrm{h}$ \\
\hline Kalijira (Thin type) & $16.60 \mathrm{a}-\mathrm{d}$ & $108.00 \mathrm{f}-\mathrm{i}$ & $13 \mathrm{ab}$ & $11 \mathrm{a}-\mathrm{d}$ & $23.67 \mathrm{~b}-\mathrm{f}$ & $78 \mathrm{f}$ & $146 \mathrm{c}-\mathrm{f}$ & $13.06 \mathrm{c}-\mathrm{e}$ & $10.9 \mathrm{~g}$ & $6.42 \mathrm{~b}$ & $2.38 \mathrm{~b}-\mathrm{f}$ \\
\hline Kalijira PL-1 & $16.20 \mathrm{a}-\mathrm{d}$ & $117.67 \mathrm{~cd}$ & $11 \mathrm{a}-\mathrm{e}$ & 10 a-e & $21.33 \mathrm{ef}$ & $81 \mathrm{~cd}$ & $151 \mathrm{c}-\mathrm{f}$ & $12.33 \mathrm{de}$ & $10.9 \mathrm{~g}$ & $6.11 \mathrm{~b}-\mathrm{f}$ & $2.44 \mathrm{~b}-\mathrm{d}$ \\
\hline Kalijira (White type) & $11.04 \mathrm{~d}-\mathrm{g}$ & $119.00 \mathrm{~cd}$ & 10 a-e & $8 b-f$ & $22.33 \mathrm{~d}-\mathrm{f}$ & $75 \mathrm{i}$ & $137 \mathrm{~d}-\mathrm{f}$ & $06.49 \mathrm{e}$ & $9.8 \mathrm{jk}$ & $5.59 \mathrm{f}$ & $2.12 \mathrm{f}-\mathrm{i}$ \\
\hline Kalijira (Late) & $12.83 \mathrm{c}-\mathrm{g}$ & $115.67 \mathrm{c}-\mathrm{f}$ & $12 \mathrm{a}-\mathrm{d}$ & $11 \mathrm{a}-\mathrm{d}$ & $25.33 \mathrm{a}-\mathrm{d}$ & $85 \mathrm{a}$ & $88 \mathrm{~g}$ & $32.69 \mathrm{a}$ & $13.6 \mathrm{c}$ & $5.69 \mathrm{ef}$ & $2.62 \mathrm{ab}$ \\
\hline Kalijira PL-6 & $16.52 \mathrm{a}-\mathrm{d}$ & 109.00 e-i & $9 \mathrm{~b}-\mathrm{e}$ & 9 a-f & $25.33 \mathrm{a}-\mathrm{d}$ & $78 \mathrm{fg}$ & $178 b c$ & $12.64 \mathrm{c}-\mathrm{e}$ & $10.4 \mathrm{~h}$ & $6.31 \mathrm{~b}-\mathrm{d}$ & $2.40 \mathrm{~b}-\mathrm{e}$ \\
\hline Kaloshailla & $18.25 \mathrm{a}-\mathrm{c}$ & $135.33 \mathrm{a}$ & $11 \mathrm{a}-\mathrm{e}$ & $11 \mathrm{a}-\mathrm{d}$ & 24.33 a-e & $73 \mathrm{j}$ & $113 f g$ & $14.90 \mathrm{~b}-\mathrm{e}$ & $15.5 \mathrm{a}$ & $7.39 \mathrm{a}$ & $2.30 \mathrm{c}-\mathrm{g}$ \\
\hline Parbatjira & $13.97 \mathrm{~b}-\mathrm{f}$ & $102.33 \mathrm{i}$ & $9 \mathrm{c}-\mathrm{e}$ & $8 \mathrm{~b}-\mathrm{f}$ & $23.33 \mathrm{c}-\mathrm{f}$ & $83 \mathrm{~b}$ & $169 \mathrm{~b}-\mathrm{d}$ & $19.75 \mathrm{~b}-\mathrm{d}$ & $9.7 \mathrm{jk}$ & $6.08 \mathrm{~b}-\mathrm{f}$ & $2.09 \mathrm{~g}-\mathrm{i}$ \\
\hline Sakkorkhora & $09.25 \mathrm{fg}$ & $92.33 \mathrm{j}$ & $13 \mathrm{ab}$ & $5 \mathrm{f}$ & $25.00 \mathrm{a}-\mathrm{d}$ & $82 \mathrm{bc}$ & $118 \mathrm{e}-\mathrm{g}$ & $25.37 \mathrm{ab}$ & $14.7 \mathrm{~b}$ & $6.94 \mathrm{a}$ & $2.76 \mathrm{a}$ \\
\hline Tilkapur & $16.98 \mathrm{a}-\mathrm{d}$ & $116.33 \mathrm{c}-\mathrm{e}$ & $14 \mathrm{a}$ & $12 \mathrm{a}$ & $23.33 \mathrm{c}-\mathrm{f}$ & $80 \mathrm{de}$ & $120 \mathrm{e}-\mathrm{g}$ & $16.05 \mathrm{~b}-\mathrm{e}$ & $11.4 \mathrm{ef}$ & $5.95 \mathrm{~b}-\mathrm{f}$ & $2.21 \mathrm{~d}-\mathrm{i}$ \\
\hline Tulshimala & $15.22 \mathrm{a}-\mathrm{f}$ & $109.33 \mathrm{e}-\mathrm{i}$ & $8 \mathrm{de}$ & $8 \mathrm{c}-\mathrm{f}$ & $26.67 \mathrm{ab}$ & $73 \mathrm{j}$ & $179 b c$ & $12.28 \mathrm{de}$ & $11.1 \mathrm{fg}$ & $6.17 \mathrm{~b}-\mathrm{f}$ & $2.4 \mathrm{~b}-\mathrm{e}$ \\
\hline Tulshimala PL-1 & $14.96 \mathrm{a}-\mathrm{f}$ & $119.67 \mathrm{bc}$ & $11 \mathrm{a}-\mathrm{e}$ & $11 \mathrm{a}-\mathrm{c}$ & $25.33 \mathrm{a}-\mathrm{d}$ & 711 & 117 e-g & $11.20 \mathrm{de}$ & $11.6 \mathrm{de}$ & $6.15 \mathrm{~b}-\mathrm{f}$ & $2.19 \mathrm{~d}-\mathrm{i}$ \\
\hline Tulshimala PL-3 & $09.55 \mathrm{e}-\mathrm{g}$ & $111.67 \mathrm{~d}-\mathrm{h}$ & $8 \mathrm{e}$ & $7 \mathrm{ef}$ & 24.33 a-e & $72 \mathrm{jk}$ & $145 \mathrm{c}-\mathrm{f}$ & $09.64 \mathrm{de}$ & $10.0 \mathrm{~h}-\mathrm{j}$ & $5.74 \mathrm{~d}-\mathrm{f}$ & $2.21 \mathrm{~d}-\mathrm{i}$ \\
\hline Minimum & 7.80 & 92 & 7 & 5 & 21 & 71 & 88 & 6.49 & 8.9 & 5.59 & 1.97 \\
\hline Maximum & 21.24 & 135 & 14 & 12 & 27.33 & 85 & 219 & 32.69 & 15.5 & 7.39 & 2.76 \\
\hline $\mathrm{LSD}_{0.05}$ & 5.38 & 6.55 & 3.49 & 3.15 & 2.59 & 1.13 & 36.59 & 9.2 & 0.3 & 0.5 & 0.22 \\
\hline
\end{tabular}
Mean followed by the same latter in a column are not significantly different from each other at $0.05 \%$ probability level. Indicators: GYP $=$ grain yield per plant
$(\mathrm{g}), \mathrm{PH}=$ plant height, TILL = tillers per plant, $\mathrm{PN}=$ panicles per plant, $50 \mathrm{~F}=$ days to $50 \%$ flowering, $\mathrm{PL}=$ panicle length, $\mathrm{G}=$ grains per panicle, $\mathrm{S}(\%)=$ grain sterility $(\%), \mathrm{TGW}=$ thousand grain weight $(\mathrm{g}), \mathrm{SL}=$ spikelet length $(\mathrm{mm})$ and $\mathrm{SB}=$ spikelet breadth 
The number of panicles per plant ranged from 5 to 12 (Table 3). Minimum number of panicles was observed in Sakkorkhora and maximum in Kalijira (Normal) and Badshabhog.

\subsection{Days to $50 \%$ flowering}

A significant variation was observed among the genotypes and coefficient of variation for days to $50 \%$ flowering was $0.88 \%$ (Table 2). Days to $50 \%$ flowering ranged from 71 to 85 days as the SGA genotypes under this study were strongly photoperiod sensitive (Table 3). The minimum days to $50 \%$ flowering were observed in Begunbichi and Tulshimala PL-1, while the maximum value was recorded in Kalijira (Late). Weiya et al. (2008) also observed variations in heading days among several genotypes and they identified a regulatory gene responsible for this variation.

\subsection{Panicle length}

Significant variation was observed in length of panicle among the genotypes at $1 \%$ levels (Table $2)$. Coefficient of variation for panicle length was $6.51 \%$ (Table 2). Panicle length displayed moderate values for coefficient of variation. Kole and Hasib, (2008) also showed the similar results. The data for panicle length ranged from 21.0 to $27.3 \mathrm{~cm}$ (Table 3 ). The minimum panicle length was recorded in Begunbichi while maximum panicle length in BR5 (Dulabhog). Ifftikhar et al. (2009) studied genetic variability for various traits and found that this trait is under the genetic control and could be used in the selection process of some desirable traits.

\subsection{Grains per panicle}

Significant genetic variation among genotypes was found for number of grains per panicle at $1 \%$ level (Table 2). Coefficient of variation for filled grains per panicle was $14.32 \%$ (Table 2). Higher coefficient of variation for filled grain was also observed by Golam et al. (2011). Number of filled grains per panicle ranged from 88 to 219 (Table 3). The least number of filled grains per panicle was observed in the genotype Kalijira (Late), while the maximum was in Chinikanai-1.

\subsection{Grain sterility (\%)}

A significant genetic variation was observed among the genotypes for the trait (Table 1). Coefficient of variation for grain sterility was $36.92 \%$ (Table 2). The grain sterility ranged from 6.49 to 32.69 (Table 3). Minimum and maximum grain sterility were observed in Kalijira (White) and in Kalijira (Late), respectively. The reason for $32.69 \%$ grain sterility of Kalijira (Late) was the prevailing low night temperature during flowering and grain filling stage.

\subsection{Thousand grain weight}

Significant variation was observed among the tested genotypes for thousand grain weight (Table 2). Coefficient of variation for 1000 grain weight was $1.83 \%$ (Table 2). The thousand grain weight ranged from $8.9 \mathrm{~g}$ to $15.5 \mathrm{~g}$ (Table 3). Minimum thousand grain weight was recorded in Kalijira (Normal), while maximum in Kaloshailla.

\subsection{Spikelet length}

Sspikelet lengths varied significantly among the genotypes (Table 2). Coefficient of variation for spikelet length was $5.00 \%$ (Table 2). Spikelet length ranged between 5.59 to $7.39 \mathrm{~mm}$ (Table 3). Minimum spikelet length was recorded in Kalijira (White type), while maximum was observed in Kaloshailla.

\subsection{Spikelet breadth}

Genetic differences were significant at $1 \%$ level among rice genotypes for spikelet breadth (Table 2). Coefficient of variation for this trait was $5.96 \%$ (Table 2). Spikelet breadth ranged from 1.97 to $2.76 \mathrm{~mm}$ (Table 3). Minimum and maximum spikelet breadth was observed in Chinigura PL-2 and Sakkorkhora, respectively.

\subsection{Grain yield per plant}

The genotypes were significantly different for this trait at 1\% level (Table 2). Coefficient of variation for grain yield per plant was $21.56 \%$ (Table 2) which indicates considerable variations among the genotypes. Higher coefficient of variation was also recorded for grain yield per 
plant (Kole and Hasib, 2008). Grain yield per plant ranged from 7.80 to $21.24 \mathrm{~g}$ (Table 3). Robin and Saha (2015) found grain yield of 8.59 $\mathrm{g}$ per plant in Kalijira genotype which was much lower than that in the present findings. Minimum grain yield per plant was recorded in Begun bichi and maximum was recorded in Chinikanai-1. Begun bichi showed least grain yield per plant due to higher spikelet sterility $(23.54 \%)$ and lower thousand grain weight $(9.6 \mathrm{~g})$.

\subsection{Comparison of agronomic characters}

SGA rice varieties showed significant differences in respect of 11 characters (Table 3). This was expected because these are landraces and are grown in different areas of Bangladesh. As compared with the check variety BRRI dhan34; Chinikanai-1, Kalijira PL-9, Kalijira PL-3 and Badshabhog were observed as the four top yielders (Table 3 ). The yield potential of these genotypes can be explained based on the larger number of panicles (Badshabhog), grains per panicle (Chinikanai-1 and Kalijira Pl-9) and increased panicle length (Chinikanai-1). Kalijira is a popular and a well accepted Bangladeshi aromatic rice variety. Comparison of agronomic characters of four selected outstanding genotypes along with standard check BRRI dhan34 is presented in Table 3.

\subsection{Correlations analysis}

Out of 11 characters, only three viz. number of tillers per plant, number of panicles per plant and grains per panicle showed positive and significant correlations with grain yields (Table 4). Positive relation of grain yield with panicles per plant was also reported by Golam et al. (2011). Negative correlation between panicles per plant and grain yield was reported by Zia-ulqamar et al. (2005), which do not support the present findings. Although significant negative correlation was observed between grain sterility (\%) and grains per panicle. Plant height had no significant correlation with grain yield. However, number of tillers per plant, panicle length, spikelet length and spikelet breadth showed positive correlation with yield. However, no significant correlation was observed between the above mentioned traits and yield in this study although their trend was positive. Golam et al. (2011) reported positive contribution of grains per panicle towards grain yield, which supports the present finding.

Table 4. Correlation coefficients among the agronomic traits using genotypes means

\begin{tabular}{lccllllllll}
\hline Traits & PH & TILL & PN & $50 F$ & PL & G & $\%$ S & HSW & SL & SB \\
\hline TILL & 0.042 & & & & & & & & & \\
PN & 0.219 & $0.616^{* *}$ & & & & & & & & \\
50F & -0.291 & 0.333 & 0.066 & & & & & & & \\
PL & 0.045 & 0.136 & 0.027 & -0.047 & & & & & & \\
G & 0.058 & -0.196 & -0.163 & 0.207 & 0.098 & & & & & \\
\%S & -0.364 & 0.03 & -0.083 & 0.316 & 0.032 & $-0.503^{*}$ & & & & \\
HSW & 0.194 & 0.295 & -0.058 & 0.029 & 0.144 & $-0.493^{*}$ & 0.369 & & & \\
SL & -0.016 & 0.231 & 0.018 & -0.096 & 0.220 & -0.171 & 0.121 & $0.632^{* *}$ & & \\
SB & -0.221 & 0.398 & 0.064 & 0.226 & 0.020 & -0.226 & 0.295 & $0.590^{* *}$ & 0.347 & \\
YIELD & 0.327 & $0.454^{*}$ & $0.646^{* *}$ & 0.19 & 0.132 & $0.525^{* *}$ & -0.377 & -0.022 & 0.165 & 0.128 \\
\hline
\end{tabular}

** indicate significant at $1 \%$ level of probability, * indicate significant at $5 \%$ level of probability, ns: not significant. Indicators: $\mathrm{GYP}=$ grain yield per plant $(\mathrm{g}), \mathrm{PH}=$ plant height, TILL=tillers per plant, $\mathrm{PN}=$ panicles per plant, $50 \mathrm{~F}=$ days to $50 \%$ flowering, $\mathrm{PL}=$ panicle length, $\mathrm{G}=$ grains per panicle, $\mathrm{S}(\%)$ =grain sterility $(\%)$, TGW=thousand grain weight $(\mathrm{g}), \mathrm{SL}=$ spikelet length and $\mathrm{SB}=$ spikelet breadth 
Table 5. Aroma score of 24 SGA genotypes based on sensory test

\begin{tabular}{lll}
\hline Genotypes & Aroma Score & Remarks \\
\hline Chinikanai-1 & 2 & Scented \\
Kalijira PL-9 & 2 & Scented \\
Kalijira PL-3 & 2 & Scented \\
Badshabhog & 2 & Scented \\
BRRI dhan34 & 2 & Scented \\
Begun bichi & 1 & Lightly scented \\
Binnaful & 1 & Lightly scented \\
BR5 (Dulabhog) & 2 & Scented \\
Chinikanai-2 & 1 & Lightly scented \\
Chinigura & 2 & Scented \\
Chinigura PL-2 & 2 & Scented \\
Kalijira (Normal) & 2 & Scented \\
Kalijira (Thin type) & 2 & Scented \\
Kalijira PL-1 & 2 & Scented \\
Kalijira (White type) & 1 & Lightly scented \\
Kalijira (Late) & 2 & Scented \\
Kalijira PL-6 & 2 & Scented \\
Kaloshailla & 1 & Lightly scented \\
Parbatjira & 1 & Lightly scented \\
Sakkorkhora & 2 & Scented \\
Tilkapur & 2 & Scented \\
Tulshimala & 2 & Scented \\
Tulshimala PL-1 & 2 & Scented \\
Tulshimala PL-3 & 2 & Scented \\
\hline
\end{tabular}

\subsection{Grain aroma}

Grain aroma test revealed that all the genotypes possessed scent. On the basis of intensity of aroma, the SGA varieties were grouped in two viz, scented and lightly scented. Within the 24 SGA genotypes, only six were lightly scented and 18 were scented (Table 5). The highest yielding four SGA genotypes - Chinikanai-1, Kalijira P1-9, Kalijira Pl-3 and Badshabhog were in scented group. Jewel et al. (2011) also found different aroma intensity in the genotypes.

\section{Conclusions}

Some of the 24 SGA rice genotypes performed better in terms of aroma score and yield related traits. Correlation analysis revealed that two agronomic traits such as number of panicles per plant (0.646) and grains per panicle (0.525) had the positive contribution to grain yield. Days to
$50 \%$ flowering, plant height, panicle length, spikelet length, and spikelet breadth had positive correlation with yield. The varieties Chinikanai1, Kalijira PL-9, Kalijira PL-3 and Badshabhog were identified as high yielders among the genotypes. The selected rice genotypes can be used as potential breeding materials to develop aromatic quality rice in Bangladesh and even for other sub-tropical countries.

\section{Acknowledgement}

The authors acknowledge Agriculture and Food Security Program of BRAC for providing funds to accomplish the research work.

\section{References}

Golam, F., Hui Yin, Y., Masitah, A., Afnierna, N., Majid, N.A., Khalid, N. and Osman, 
M. 2011. Analysis of aroma and yield components of aromatic rice in Malaysian tropical environment. Australian Journal of Crop Sciences, 5(11): 1318-1325.

Ifftikhar, J., Khalil, H., Abdul, B., Sajid, K., and Islam, Z. 2009. Genetic variation for yield and yield components in rice. $A R P N$ Journal of Agricultural and Biological Science, 4 (6): 60-64.

IRFAON. 2012. Thirteenth International Fine Grain Aromatic Rice Observational Nursery Field Book. International Rice Research Institute, 2 p.

IRRI (International Rice Research Institute). 2002. Standard Evaluation System for rice (SES), $4^{\text {th }}$ edition. Los Banos (Philippines), International Rice Research Institute, $31 \mathrm{p}$.

Jewel, Z. A., Patwary, A. K., Maniruzzaman, S., Barua, R. and Begum, S. N. 2011. Physico-chemical and Genetic Analysis of Aromatic Rice (Oryza sativa L.) Germplasm, The Agriculturists, 9(1\&2): 82-88.

Kole, P. C. and Hasib, K. M. 2008. Correlation and regression analysis in scented rice. Madras Agricultural Journal, 95(1-6): 178-182.
Robin, A. H. K. and Saha, P. S. 2015 Morphology of lateral roots of twelve rice cultivars of Bangladesh: dimension increase and diameter reduction in progressive root branching at the vegetative stage. Plant Root, 9: 34-42.

Tahir, M., Wandan, D. and Zada, A. 2002. Genetic variability of different plant yield characters in rice. Sarhad Journal of Agriculture, 18(2): 22-27

Utz, H. F. 2007. PLABSTAT (Version 2N). A computer program for the computation of variances and co-variances. Institute of Plant Breeding, Seed Science and Population Genetics, University of Hohenheim, Stuttgart, Germany.

Weiya, X., Yongzhong, X., Xiaoyu, W., Yu, Z., Weijiang, T., Lei, W. and Hongju, $\mathrm{Z}$. 2008. Natural variation in Ghd7 is an important regulator of heading date and yield potential in rice, Nature Genetics, 40: 761 - 767.

Zia-ul-qamar A., Cheema A., Ashraf M. and Tahir G.R. 2005. Association analysis of some yield influencing traits in aromatic and non-aromatic rice. Pakistan Journal of Botany, 37(3): 613-627. 